\title{
Influence of motor impairment on exercise capacity and quality of life in patients with Parkinson disease
}

\author{
Hélcio Kanegusuku1,*, Raphael M. Ritti-Dias ${ }^{2}$, Pâmela Yuki Igarasi Barbosaa, ${ }^{3,4}$ Erica Tardelli das Neves Guelfi ${ }^{4}$, Erika Okamoto ${ }^{4}$, \\ Camila Souza Miranda ${ }^{3}$, Tatiana de Paula Oliveira ${ }^{3}$, Maria Elisa Pimentel Piemonte ${ }^{3}$ \\ ${ }^{1}$ Hospital Israelita Albert Einstein, São Paulo, Brazil \\ ${ }^{2}$ Graduated Program in Rehabilitation Sciences, Universidade Nove de Julho, São Paulo, Brazil \\ ${ }^{3}$ Faculty of Medical Science, University of São Paulo, São Paulo, Brazil \\ ${ }^{4}$ Brazil Parkinson Association, São Paulo, Brazil
}

This study evaluated the impact of motor impairment (MI) on exercise capacity and quality of life in patients with Parkinson disease (PD). One hundred ninety-two patients ( $\geq 50$ years old) were divided according to the Hoehn and Yahr stages in: mild (stage I), mild to moderate (stage II), moderate (stage III), and advanced MI (stage IV). Exercise capacity (6-min walk test [6MWT]) and quality of life (Parkinson's Disease Questionnaire [PDQ-39]) were obtained. In this context, 6MWT was progressively worse with increasing the severity of $\mathrm{MI}(P<0.01)$. Patients with advanced $\mathrm{MI}$ achieved $39 \%$ of predicted $6 \mathrm{MWT}$ of healthy subject, while subjects with mild $\mathrm{Ml}$ achieved $83 \%$ of healthy subject $(P<0.01)$. In addition, patients with advanced MI presented higher (i.e., worse) PDQ-39 scores in summary index, cognition, mobility and activities of daily live domains compared to other groups $(P<0.01)$. Patients with moderate $\mathrm{MI}$ also presented worse scores in PDQ-39 summary index, mobility and activities of daily live domains in comparison with mild $\mathrm{MI}$ patients $(P<0.01)$. Higher MI was correlated with worse exercise capacity (6MWT: $r=-0.46, P<0.01$ ), with worse PDQ-39 summary index and the mobility and activities of daily live domains scores $(r=0.38, r=0.46$, and $r=0.43, P<0.01)$. In conclusion, $\mathrm{Ml}$ is related to lower exercise capacity and quality of life (i.e., PDQ-39 summary index and mobility and activities of daily live domains) in patients with PD.

Keywords: Parkinsonism, Functional capacity, Motor impairment, Quality of life.

\section{INTRODUCTION}

In the last 25 years, the prevalence of people with Parkinson disease (PD) worldwide more than double from 2.5 million to 6.1 million (GBD 2016 Parkinson's Disease Collaborators, 2018). PD is a progressive neurodegenerative disease characterized by motor impairment (MI), involving resting tremor, rigidity, bradykinesia, and postural instability, affecting the mobility (Dickson, 2018; Jankovic, 2008) and reduced survival in these patients (Lo et al., 2009).

The Hoehn and Yahr scale (HY) is the most common applied tool for evaluate the level of clinical disability in these patients. As an indicator of PD progression, change in HY stage (i.e., stages $1-5$ : mild-severe levels) is an important feature in the course of the PD (Goetz et al., 2004). In this context, previous studies (Falvo and Earhart, 2009; Ivey et al., 2012) observed relationship between worse exercise capacity (6-min walk test [6MWT]) and higher MI assessed by the HY scale in patients with mild to moderate PD (i.e., stages 1-3). Despite these results, studies including patients in more advanced stage of $\mathrm{PD}$ are still needed for a better understanding of this relationship.

Balzer-Geldsetzer et al. (2018) also observed relationship between higher MI assessed by the HY scale and worse global quality of life as well as in some of the domains of quality of life (i.e., mobility, activities of daily live, emotional well-being, cognition, and communication domains) in patients with mild to moderate PD. However, although previous studies (He et al., 2016; Rosqvist et al., 2021) have also observed relationship between higher MI
${ }^{*}$ Corresponding author: Hélcio Kanegusuku (D) https://orcid.org/0000-0002-0406-6504 Hospital Israelita Albert Einstein, Av. Albert Einstein, 627/701, São Paulo 05651-901, Brazil

Email: helciokng@gmail.com

Received: May 15, 2021 / Accepted: June 18, 2021
This is an Open Access article distributed under the terms of the Creative Commons Attribution Non-Commercial License (https://creativecommons.org/licenses/by-nc/4.0/) which permits unrestricted non-commercial use, distribution, and reproduction in any medium, provided the original work is properly cited. 
and worse global quality of life in patients with advanced PD, they did not assess the different domains of quality of life. So, the impact of greater MI severity on the different domains of quality of life is unclear.

The gap finding regarding the role of MI severity accessed by the HY scale on exercise capacity and quality of life can be caused by limited characteristics of the sample studied (i.e., HY stages 1-3), limiting the understanding the consequences of $\mathrm{PD}$ on $\mathrm{MI}$ severity spectrum. Therefore, in the aim of the present study was to analyze in patients with PD with severity ranging from $\mathrm{HY}$ stage 1 to 4 the relationship between MI on exercise capacity and quality of life.

\section{MATERIALS AND METHODS}

\section{Design}

This study has a cross-sectional study performed in a single center between October 2016 and April 2019. For data analyses, patients with PD were divided according to the HY stages in: mild (stage I), mild to moderate (stage II), moderate (stage III), and advanced MI (stage IV). Sociodemographic and clinical information was obtained through a face-to-face interview. In addition, exercise capacity and quality of life were also assessed. All the evaluations were performed with patients in the "on state" of their medication of PD.

\section{Subjects}

Patients with PD were recruited from The Brazil Parkinson Association. Patients were included if they met the following criteria: age $\geq 50$ years old; diagnosis of PD; and stages I-IV of the HY. Exclusion criteria included: presence of any health problem that preclude tests. The study was approved by the local ethical committee (CAAE: 15050713.6.2004.0065), according to the Declaration of Helsinki, and written informed consent was obtained from the patients.

\section{Clinical evaluation}

The patients' clinical stages were assessed using the HY scale, raging I (mild problem) to stage $\mathrm{V}$ (advanced disease) (Goetz et al., 2004). Their IM was also assessed using the Unified Parkinson's Disease Rating Scale (UPDRS) part III (Goetz et al., 2008). It consists of 27 items (bradikynesia, rigidity, tremor, and axial signs), ranging 0 (no problem) to 4 points (severe problem). Levodopa equivalent dose (LED) was calculated using a previously published formula (Tomlinson et al., 2010).

\section{Exercise capacity evaluation}

The 6MWT was performed along a 30-m corridor. A cone was placed at each end of the walkway to indicate where the patient had to turn. Patients were instructed to cover as much distance as possible in 6 min (ATS Committee on Proficiency Standards for Clinical Pulmonary Function Laboratories, 2002). Six-min total distance was defined as the maximum distance achieved by the patient at the end of the test. The percentage of predicted 6MWT of a healthy subject with similar characteristics was calculated using the procedures previously described (Iwama et al., 2009).

\section{Quality of life}

Quality of life was assessed using the validated Brazilian version of the Parkinson's Disease Questionnaire (PDQ-39) (Carod-Artal et al., 2007). Each of its 39 questions uses the 5 points ordinal scoring system. It has eight domains: mobility, activities of daily live, emotional well-being, stigma, social support, cognition, communication, and bodily discomfort. The questions are presented below:

Mobility: 1. Had difficulty doing leisure activities? 2. Had difficulty looking after your home? 3. Had difficulty carrying shopping bags? 4. Had difficulty walking half a mile? 5. Had difficulty walking 100 yards? 6 . Had difficulty getting around the house? 7. Had difficulty getting around in public places? 8 . Needed to be accompanied when went out? 9. Felt frightened or worried about falling in public? 10. Been confined to the house more than liked?

Activities of daily live: 1 . Had difficulty washing yourself? 2 . Had difficulty dressing yourself? 3. Had problems doing up buttons or shoe laces? 4. Had problems writing clearly? 5. Had difficulty cutting up your food? 6. Had difficulty holding a drink?

Emotional well-being: 1. Felt depressed? 2. Felt isolated and lonely? 3. Felt weepy or tearful? 4. Felt angry or bitter? 5. Felt anxious? 6. Felt worried about your future?

Stigma: 1. Felt you had to conceal PD? 2. Avoided eating or drinking in public? 3. Felt embarrassed about having PD? 4. Felt worried about other people's reaction to you?

Social support: 1. Had problems with close relationships? 2. Lacked support from your spouse or partner? 3. Lacked support from friends' family or close friends?

Cognition: 1. Unexpectedly fallen asleep during the day? 2. Had problems with concentration? 3. Felt your memory was bad? 4 . Had distressing dreams or hallucinations?

Communications: 1 . Had difficulty with speech? 2. Felt unable to communicate properly? 3. Felt ignored by people?

Bodily discomfort: 1. Had muscle cramps or spasms? 2. Had 
aches or pains? 3. Felt unpleasantly hot or cold?

Answers for each of the questions range from 0 (never) to 4 (always). Subscale scores and a summary index representing the quality of life may also be calculated (0-100), with higher scores representing worse quality of life (Carod-Artal et al., 2007).

\section{Statistical analyses}

The chi-square was used for comparing differences among the groups regarding categorical variables. Generalized linear model was used for continuous variables. Correlation between the HY and functional capacity and the different domains of quality of life was analyzed by Partial correlation. In both analyzes, age, gender, disease duration, and LED were employed as covariate. Post hoc pairwise comparison using the Bonferroni correction for multiple comparisons was employed when necessary. All statistical analysis was performed using IBM SPSS Statistics ver. 20.0 (IBM Co., Armonk, NY, USA). Significance level was defined as $P \leq 0.05$.

\section{RESULTS}

One-hundred ninety-two patients with PD were included in the study, being 26 with mild MI, 51 patients with mild to moderate MI, 92 patients with moderate MI, and 23 patients with advanced MI. Their characteristics are shown in Table 1. Groups were similar regarding body mass index, comorbidities, and medications for other comorbidities. However, patients with moderate MI presented difference for the gender proportion compared to the other patients. Patients with advanced MI were older and had longer disease duration compared to other patients. Patients with mild to moderate and moderate MI were older and had longer disease duration compared to patients with mild MI. Patients with more MI also presented higher UPDRS part III score compared to the other patients. In addition, patients with moderate and advanced MI had a higher LED compared to the other patients.

Patients with advanced MI had worse performance on 6MWT

Table 1. Characteristics of the patients with Parkinson disease divided according to motor impairment $(n=192)$

\begin{tabular}{|c|c|c|c|c|c|}
\hline Characteristic & Mild $(n=26)$ & Mild to moderate $(n=51)$ & Moderate $(\mathrm{n}=92)$ & Advanced ( $n=23)$ & $P$-value \\
\hline \multicolumn{6}{|l|}{ Physical characteristics } \\
\hline Gender, male:female (n) & $18: 8^{\ddagger}$ & $39: 12^{\ddagger}$ & $48: 44$ & $10: 13^{\ddagger}$ & $<0.01$ \\
\hline Age (yr) & $63 \pm 2$ & $69 \pm 1^{*}$ & $70 \pm 1^{*}$ & $75 \pm 2^{*,+, \pm}$ & $<0.01$ \\
\hline Body mass index (kg/m²) & $26.7 \pm 1.0$ & $25.7 \pm 0.6$ & $27.0 \pm 0.5$ & $25.4 \pm 1.1$ & 0.31 \\
\hline \multicolumn{6}{|l|}{ Parkinson disease characteristics } \\
\hline Disease duration (yr) & $3.3 \pm 0.9$ & $5.7 \pm 0.6^{*}$ & $5.5 \pm 0.5^{*}$ & $8.7 \pm 1.0^{*}, t, \pm$ & $<0.01$ \\
\hline UPDRS part III (score) & $12 \pm 2$ & $20 \pm 1^{*}$ & $28 \pm 1^{*,+}$ & $43 \pm 2^{*, t, \pm}$ & $<0.01$ \\
\hline LED (mg/day) & $301 \pm 50$ & $419 \pm 35$ & $476 \pm 26^{*}$ & $517 \pm 53^{*}$ & $<0.01$ \\
\hline \multicolumn{6}{|l|}{ Comorbid conditions (\%) } \\
\hline Hypertension & 30.8 & 37.3 & 47.8 & 47.8 & 0.34 \\
\hline Dyslipidemia & 19.2 & 25.5 & 26.1 & 21.7 & 0.89 \\
\hline Diabetes Mellitus & 11.5 & 15.7 & 25.0 & 30.4 & 0.23 \\
\hline Cardiac disease & 15.4 & 9.8 & 13.1 & 21.7 & 0.57 \\
\hline Depression & 15.4 & 21.6 & 19.6 & 26.1 & 0.81 \\
\hline Bone diseases & 11.5 & 15.7 & 29.3 & 30.4 & 0.10 \\
\hline \multicolumn{6}{|c|}{ Medications for comorbid conditions (\%) } \\
\hline Beta blockers & 23.1 & 13.7 & 15.2 & 17.4 & 0.75 \\
\hline Calcium channel blockers & 0.0 & 3.9 & 8.7 & 4.3 & 0.33 \\
\hline Inhibitor of ACE & 11.5 & 7.8 & 16.3 & 17.4 & 0.50 \\
\hline Angiotensin-receptor antagonist & 3.8 & 17.6 & 19.6 & 13.0 & 0.27 \\
\hline Vasodilator & 0.0 & 3.9 & 4.3 & 0.0 & 0.54 \\
\hline Lipid lowering & 15.4 & 21.6 & 21.7 & 21.7 & 0.91 \\
\hline Antidiabetics & 7.7 & 11.8 & 16.3 & 21.7 & 0.48 \\
\hline Antidepressants & 15.4 & 21.6 & 19.6 & 26.1 & 0.81 \\
\hline
\end{tabular}

Values are presented as mean \pm standard error unless otherwise indicated.

UPDRS, Unified Parkinson's Disease Rating Scale; LED, Levodopa equivalent dose; ACE, angiotensin-converting enzyme.

${ }^{*}$ Significantly different from mild motor impairment $(P \leq 0.05)$. ${ }^{\dagger}$ Significantly different from stage mild to moderate motor impairment $(P \leq 0.05)$. ${ }^{\ddagger}$ Significantly different from moderate motor impairment $(P \leq 0.05)$. 
Table 2. Exercise capacity of patients with Parkinson disease divided according to motor impairment

\begin{tabular}{lrrrrr}
\hline Function capacity & \multicolumn{1}{c}{ Mild } & Mild to moderate & Moderate & Advanced & $P$-value \\
\hline 6-min walk $(m)$ & $441 \pm 21(n=25)$ & $410 \pm 14(n=51)$ & $349 \pm 10^{*,+}(n=91)$ & $216 \pm 22^{*,+, \pm}(n=22)$ & $<0.01$ \\
\% of predicted the 6-min walk in healthy subjects $(m)$ & $83 \pm 4(n=25)$ & $77 \pm 3(n=51)$ & $65 \pm 2^{*,+}(n=91)$ & $39 \pm 4^{*,+, \pm}(n=22)$ & $<0.01$ \\
\hline
\end{tabular}

Values are presented as mean \pm standard error.

Generalized linear model adjusted for age, sex, disease duration, and levodopa equivalent dose.

${ }^{*}$ Significantly different from mild motor impairment $(P \leq 0.05)$. ' Significantly different from stage mild to moderate motor impairment $(P \leq 0.05)$. ${ }^{\ddagger}$ Significantly different from moderate motor impairment $(P \leq 0.05)$.

Table 3. Scores corresponding the different domains of quality of life of patients with Parkinson disease divided according to motor impairment

\begin{tabular}{lccccr}
\hline Domain & $\begin{array}{c}\text { Mild } \\
(\mathrm{n}=25)\end{array}$ & $\begin{array}{c}\text { Mild to } \\
\text { moderate } \\
(\mathrm{n}=51)\end{array}$ & $\begin{array}{c}\text { Moderate } \\
(\mathrm{n}=89)\end{array}$ & $\begin{array}{c}\text { Advanced } \\
(\mathrm{n}=22)\end{array}$ & $P$-value \\
\hline Mobility & $19 \pm 4$ & $23 \pm 3$ & $38 \pm 2^{*,+}$ & $65 \pm 5^{*,+ \pm \pm}$ & $<0.01$ \\
Activities of daily live & $25 \pm 4$ & $30 \pm 3$ & $39 \pm 3^{*}$ & $67 \pm 10^{*,+ \pm}$ & $<0.01$ \\
Emtional well-being & $25 \pm 5$ & $27 \pm 3$ & $32 \pm 2$ & $41 \pm 5$ & 0.16 \\
Stigma & $23 \pm 5$ & $31 \pm 5$ & $26 \pm 3$ & $27 \pm 6$ & 0.67 \\
Social support & $17 \pm 4$ & $19 \pm 2$ & $28 \pm 3$ & $26 \pm 5$ & 0.05 \\
Cognition & $18 \pm 4$ & $26 \pm 3$ & $26 \pm 2$ & $45 \pm 4^{*,+ \pm}$ & $<0.01$ \\
Communication & $20 \pm 4$ & $30 \pm 3$ & $28 \pm 2$ & $47 \pm 7^{*}$ & 0.01 \\
Bodily discomfort & $29 \pm 5$ & $32 \pm 3$ & $35 \pm 2$ & $45 \pm 5$ & 0.45 \\
PDQ-39 summary index & $17 \pm 2$ & $22 \pm 2$ & $26 \pm 2^{*}$ & $46 \pm 6^{*,+ \pm}$ & $<0.01$ \\
\hline
\end{tabular}

Values are presented as mean \pm standard error. Generalized linear model adjusted for age, sex, disease duration, and levodopa equivalent dose.

PDQ-39, Parkinson's Disease Questionnaire.

*Significantly different from mild motor impairment $(P \leq 0.05)$. ${ }^{~}$ Significantly different from stage mild to moderate motor impairment $(P \leq 0.05)$. "Significantly different from moderate motor impairment $(P \leq 0.05)$.

compared to other patients. Patients with moderate MI were also worse compared to patients with mild and mild to moderate MI. Patients with advanced MI achieved 39\% of predicted 6MWT of a healthy subject, while subjects with moderate MI achieved 65\% and subjects with mild to moderate and mild achieved $77 \%$ and $83 \%$ of a healthy subject, respectively (Table 2).

Patients with advanced MI had worse scores in PDQ-39 summary index and mobility, activities of daily live, and cognition domains compared to other groups. Patients with moderate MI also presented worse scores in mobility domain in comparison with mild and mild to moderate MI patients and worse scores in PDQ-39 summary index and activities of daily live domain in comparison with mild patients. There was no difference between patients with mild and mild to moderate MI (Table 3).

Higher MI correlated with worse exercise capacity (6MWT: $r=-0.46 ; \%$ of predicted the 6MWT in healthy subjects: $r=-0.47$, $P<0.01)$. In addition, except for stigma domain $(r=-0.02, P \geq 0.05)$, higher MI correlated with worse scores in all other domains of quality of life (i.e., PDQ-39 summary index, $r=0.38$; mobility, $r=0.46$; activities of daily live, $r=0.43$; emotional well-being, $r=0.17$; social support, $r=0.21$; cognition, $r=0.24$; communication, $r=0.30$; and bodily discomfort domains, $r=0.15 ; P \leq 0.05$ ).

\section{DISCUSSION}

The main findings of the current study were that the severity of MI assessed by the HY scale is related to lower exercise capacity and quality of life (i.e., PDQ-39 summary index and mobility, activities of daily live and cognition domains) in patients with PD.

The results of the current study indicated that higher MI occurred in patients older and with longer disease duration. Aging and disease duration are independent risk factors for motor symptoms in these patients. Specifically, disease duration, this is consistent with the natural history of PD as a progressive neurodegenerative disease, where MI progress throughout the course of disease (Hattori et al., 2014). However, although the relationship between aging and MI be consensus, the mechanisms surrounding the aging process on PD are unclear (Obeso et al., 2010). It has been assumed that the aging process increases the vulnerability and loss of dopaminergic neurons to toxic insult because of increasing failure of normal cellular physiological and biochemical processes and, consequently, impairment motor control of these patients (Schapira and Jenner, 2011).

Patients with higher MI had more difficulty to complete 6MWT. In fact, HY stages were moderately correlated with worse exercise capacity. These results are in accordance with a Falvo and Earhart (2009) that also observed that higher MI is associated with lower 6MWT and with Barbieri et al. (2012) that observed association with lower 880-yd walk test performance in patients with mild to moderate disease severity. Higher HY stage reflects in a worse bradykinesia, rigidity, tremor, and instability postural (Goetz et al., 2004) and, consequently, limits the tasks evaluated during 6MWT. In fact, previous studies (Falvo and Earhart, 2009; Kobayashi et al., 2017) demonstrated that 6MWT is associated with walk speed, balance, and agility. In addition, patients with advanced 
MI achieved only $39 \%$ of a healthy subject, while patients with moderate MI achieved 65\%, patients with mild to moderate MI achieved $77 \%$ and patients with mild MI achieved $83 \%$ of a healthy subject. These responses are similar to other diseases in which patients have major motor limitations, such as intermittent claudication and stroke (Correia et al., 2020; Pradon et al., 2013). Interestingly, the lower exercise capacity in patients with more severe MI occurred despite the higher LED use in these patients, which suggests that drug treatment is not completely effective in controlling MI from PD.

In this current study, advanced MI resulted in worse mobility, activities of daily live, and cognition domains, consequently, quality of life PDQ-39 summary index. In addition, moderate MI also resulted worse mobility, activities of daily live domains and, consequently, quality of life PDQ-39 summary index compared to patients with mild MI. In fact, HY stages were moderately correlated with worse the mobility and activities of daily live domains and, consequently, PDQ-39 summary index of quality of life. Balzer-Geldsetzer et al. (2018) also observed that moderate MI resulted worse mobility and activities of daily live domains and quality of life PDQ-39 summary index compared with patients with less MI. Higher MI results in more difficulty for patients to perform tasks related mobility (e.g., walking) and activities of daily live (e.g., dressing, eating, etc.), parameters encompassed by HY stages (i.e., bilateral involvement, postural instability, and severe disability but still able to walk or stand unassisted) (Goetz et al., 2004) obtained in this current study. Regarding cognition domains result, higher MI has been associated with reduction in the physical activity level in patients with PD (Bryant et al., 2016), contributing, consequently, with impairment on cognitive capacity (Shih et al., 2019).

The results of the current study have some practical relevance, since it was possible to observe that the severity of MI assessed by the HY scale is related to lower exercise capacity and quality of life in patients with PD. In practical terms, the HY scale is a practical and fast scale designed to assess the MI and understanding the relationship between the severity of MI and exercise capacity and quality of life may allow clinicians and others rehabilitation professionals to better quantify physical functioning and quality of life, longitudinally assess disease progression, and also assess the efficacy of interventions. Furthermore, the results emphasize the importance of optimizing treatment for patients with more advanced MI.

This study had some limitations. (a) It is a cross-sectional study and so cannot establish cause and effect; (b) Patients at the HY stage 5 were excluded of this study. Thus, results cannot be generalized to all patients with PD. However, the inclusion of these patients would not guarantee the correct execution of the tests and questionnaires applied in study due their motor and non-motor impairments; (c) Results are limited the tests and questionnaires applied in this study and may be different of other tests, which should be tested.

In conclusion, MI is related to lower exercise capacity and quality of life (i.e., PDQ-39 summary index and mobility, activities of daily live and cognition domains) in patients with PD.

\section{CONFLICT OF INTEREST}

No potential conflict of interest relevant to this article was reported.

\section{ACKNOWLEDGMENTS}

Fundação de Amparo à Pesquisa do Estado de São Paulo FAPESP' (2013/07699-0). RMR receive a research productivity fellowship granted by Brazilian National Council for Scientific and Technological Development. We gratefully acknowledge the volunteers and The Brazil Parkinson Association and the Research, Innovation and Dissemination Center for Neuromathematics (NeuroMat).

\section{REFERENCES}

ATS Committee on Proficiency Standards for Clinical Pulmonary Function Laboratories. ATS statement: guidelines for the six-minute walk test. Am J Respir Crit Care Med 2002;166:111-117.

Balzer-Geldsetzer M, Klotsche J, LANDSCAPE Consortium, Dorel R, Riedel O. Quality of life in a German cohort of Parkinson's patients assessed with three different measures. J Neurol 2018;265:2713-2722.

Barbieri FA, Rinaldi NM, Santos PCR, Lirani-Silva E, Vitório R, TeixeiraArroyo C, Stella F, Gobbi LT, PROPARKI Group. Functional capacity of Brazilian patients with Parkinson's disease (PD): relationship between clinical characteristics and disease severity. Arch Gerontol Geriatr 2012;54:83-88.

Bryant MS, Hou JG, Coolins RL, Protas EJ. Contribution axial motor impairment to physical inactivity in Parkinson disease. Am J Phy Med Rehabil 2016;95:348-354.

Carod-Artal FJ, Martinez-Martin P, Vargas AP. Independent validation of SCOPA-psychosocial and metric properties of the PDQ-39 Brazilian version. Mov Disord 2007;22:91-98. 
Correia MA, de Sousa ASA, Andrade-Lima A, Germano-Soares AH, Zerati AE, Puech-Leão P, Wolosker N, Gardner AW, Cucato GG. Functional and cardiovascular measurements in patients with peripheral artery disease: comparison between men and women. J Cardiopulm Rehabil Prev 2020;40:24-28.

Dickson DW. Neuropathology of Parkinson disease. Parkinsonism Relat Disord 2018;46(Suppl 1):S30-S33.

Falvo MJ, Earhart GM. Six-minute walk distance in persons with Parkinson disease: a hierarchical regression model. Arch Phys Med Rehabil 2009;90:1004-1008.

GBD 2016 Parkinson's Disease Collaborators. Global, regional, and national burden of Parkinson's Disease, 1990-2016: systematic analysis for the Global Burden of Disease Study 2016. Lancet Neurol 2018;17: 939-953.

Goetz CG, Poewe W, Rascol O, Sampaio C, Stebbins GT, Counsell C, Giladi N, Holloway RG, Moore CG, Wenning GK, Yahr MD, Seidl L, Movement Disorder Society Task Force on Rating Scales for Parkinson's Disease. Movement Disorder Society Task Force report on the Hoehn and Yahr staging scale: status and recommendations. Mov Disord 2004;19:1020-1028.

Goetz CG, Tilley BC, Shaftman SR, Stebbins GT, Fahn S, Martinez-Martin P, Poewe W, Sampaio C, Stern MB, Dodel R, Dubois B, Holloway R, Jankovic J, Kulisevsky J, Lang AE, Lees A, Leurgans S, LeWitt PA, Nyenhuis D, Olanow CW, Rascol O, Schrag A, Teresi JA, van Hilten JJ, LaPelle N, Movement Disorder Society UPDRS Revision Task Force. Movement Disorder Society-sponsored revisio of The Unified Parkinson's Disease Rating Scale (MDS-UPDRS): scale presentation and clinimetric testing results. Mov Disord 2008;23:2129-2170.

Hattori T, Orimo S, Hallett M, Wu T, Inaba A, Azuma R, Mizusawa H. Relationship and factor structure in multisystem neurodegeneration in Parkinson's disease. Acta Neurol Scand 2014;130:347-353.

He L, Lee E, Sterling NW, Kong L, Lewis MM, Du G, Eslinger PJ, Huang $X$. The key determinants to quality of life in Parkinsin's disease patients: results from the Parkinson's disease biomarker program (PDBP). J Parkins Dis 2016;6:523-532.
Ivey FM, Katzel LI, Sorkin JD, Macko RF, Shulman LM. The Unified Parkinson's Disease Rating Scale as a predictor of peak aerobic capacity and ambulatory function. J Rehabil Res Dev 2012;49:1269-1276.

Iwama AM, Andrade GN, Shima P, Tanni SE, Godoy I, Dourado VZ. The six-minute walk test and body weight-walk distance product in healthy Brazilian subjects. Braz J Med Biol Res 2009;42:1080-1085.

Jankovic J. Parkinson's disease: clinical features and diagnosis. J Neurol Neurosurg Psychiatry 2008;79:368-376.

Kobayashi E, Himuro N, Takahashi M. Clinical utility of the 6-min walk test for patients with moderate Parkinson's disease. Int J Rehabil Res 2017;40:66-70.

Lo RY, Tanner CM, Albers KB, Leimpeter AD, Fross RD, Bernstein AL, McGuire V, Quesenberry CP, Nelson LM, Van Den Eeden SK. Clinical features in early Parkinson disease and survival. Arch Neurol 2009;66: 1353-1358.

Obeso JA, Rodriguez-Oroz MC, Goetz CG, Marin C, Kordower JH, Rodriguez M, Hirsch EC, Farrer M, Schapira AH, Hallidat G. Missing pieces in the Parkinson's disease puzzle. Nat Med 2010;16:653-661.

Pradon D, Roche N, Enette L, Zory R. Relationship between lower limb muscle strength and 6-minute walk test performance in stroke patients. J Rehabil Med 2013;45:105-108.

Rosqvist K, Odin P, Lorenzl S, Meissner WG, Bloem BR, Ferreira JJ, Dodel R, Schrag A; Care of Late Stage Parkinsonism (CLaSP) Consortium. Factors associated with health-related quality of life in late-stage Parkinson's disease. Mov Disord Clin Pract 2021;8:563-570.

Schapira AH, Jenner P. Etiology and pathogenesis of Parkinson's disease. Mov Disord 2011;26:1049-1055.

Shih CH, Moore K, Browner N, Sklerov M, Dayan E. Physical activity mediates the association between striatal dopamine transporter availability and cognition in Parkinson's disease. Parkinsonism Relat Disord 2019;62:68-72.

Tomlinson CL, Stowe R, Patel S, Rick C, Gray R, Clarke CE. Systematic review of levodopa dose equivalency reporting in Parkinson's disease. Mov Disord 2010;25:2649-2653. 